\title{
Formal Specification and Analysis of Termination Detection by Weight-throwing Protocol
}

\author{
Imran Riaz Hasrat, Muhammad Atif \\ Department of Computer Science \\ and Information Technology \\ The University of Lahore \\ Lahore, Pakistan
}

\author{
Muhammad Naeem \\ Department of Electronics \\ and Electrical Systems \\ The University of Lahore \\ Lahore, Pakistan
}

\begin{abstract}
Termination detection is a critical problem in distributed systems. A distributed computation is called terminated if all of its processes become idle and there are no in-transit messages in communication channels. A distributed termination detection protocol is used to detect the state of a process at any time, i.e., terminated, idle or active. A termination detection protocol on the basis of weight-throwing scheme is described in Yu-Chee Tseng, "Detecting Termination by Weight-throwing in a Faulty Distributed System", JPDC, 15 February 1995. We apply model checking techniques to verify the protocol and for formal specification and verification the tool-set UPPAAL is used. Our results show that the protocol fails to fulfil some of its functional requirements.
\end{abstract}

Keywords-Termination detection; weight-throwing protocol; formal specification and verification; model checking

\section{INTRODUCTION}

Termination detection is an important problem for distributed systems. For a distributed system, termination detection is based on the concept of a process state. During a distributed computation, a process can either be in alive or dead state. An alive state means that a process is still performing its task whereas dead state represents that the process becomes idle simultaneously. Dead and alive states are referred as passive and active states as shown in Fig. 1. At the start of the computation, all the processes are supposed to be in active state. Processes can take several actions discussed below:

- Only the processes in active state can send basic messages to other processes.

- Any active process can reach a passive state at any time.

- A passive process becomes active again by receiving a basic message.

A distributed computation is called terminated if all of its processes become passive and there are no in-transit messages in the communication channels.

Many applications of distributed systems depend on termination detection of a computation to guarantee a proper operation. In multiphase algorithms [2], one phase depends on proper completion of other phase. So, initiation of new phase needs termination detection of previous phase. In distributed databases, deadlock detection is a critical problem and this

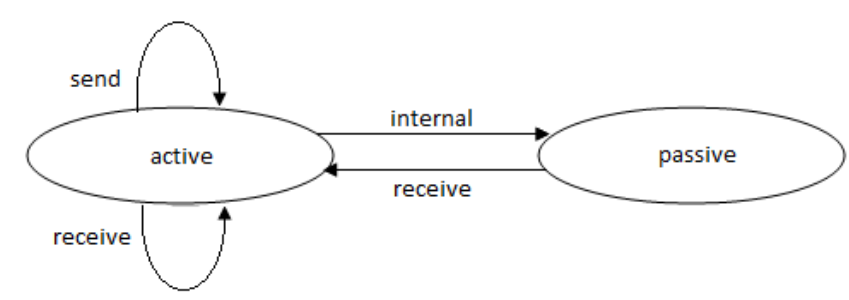

Fig. 1. Termination detection [1].

problem is purely related to termination detection [3]. Garbage collection [4] and token loss detection in a token ring are other examples of termination detection problems. Termination detection solution allows a system to guarantee that all tasks in the system are obviously complete and this permits the dependent systems to start their computations. About more than three decades ago, termination detection problem was separately suggested by Dijkstra and Scholten [5] and Francez [6]. Many researchers started to tackle this problem by developing different termination detection algorithms described in [7]-[15].

Formal methods are based on mathematical tools and techniques used for design, specification and verification of different hardware and software systems. Formal methods provide correctness for all requirements and inputs of a given system. In the past, formal methods were used for only safety critical and defense related systems [16]-[18]. Now a days, high demand of error free and secure systems is giving much importance to formal methods.

We present a formal modeling and analysis of the termination detection by weight-throwing in a faulty distributed system presented in [7]. It is a comprehensive analysis of all possible versions of protocol along with verification of detailed functional requirements. Basic concept in weightthrowing protocol is that each process sends some weight with every basic message. On reception of this message, recipient process adds this weight to its current weight. We present formal verification of the weight-throwing protocol using a model checker known as UPPAAL.

UPPAAL has a simulator that is used to develop the model [19]. The verifier in UPPAAL has capability to create the traces that can lead the action sequences where system's required 
property fails. To investigate this situation, action sequences are replayed in simulator.

We formally model and analyze both parts of the protocol. We present a formal specification of the protocol in the timedautomata language of UPPAAL. Then, we specify functional requirements for safety and liveness of the protocol. We also present specification and verification of its invariants. We also analyze the protocol in the situations when some processes may tend to fail. We discover some situations in which protocol fails to satisfy its functional requirements, termination is not detected in these situations. The protocol also fails to satisfy some of its invariants. We present counterexamples for the requirement violations in the form of message sequence charts.

\section{Weight-Throwing Protocol}

Weight-throwing protocol works in a distributed system. In this protocol a process sends half of its weight with its message when it communicates to some other process. Let $S$ is the set of processes i.e. $S=\left\{P_{1}, P_{2}, \cdots, P_{n}\right\}$. The $S$ is supposed to be fault-free. In fault-free system, a process never fails. A process from $S$ with minimum id is said to be the leader. Total weight in the system is 1 . Every process is active at the start of the computation and weight of each process $P_{i}$ is $w_{i}=1 / n$, where $n$ is the cardinality of the set $S$. The leader collects all the weights in the system and announce termination. Upon every message from a process $P_{i}$ to $P_{j}$ following actions are performed:

1) $\quad P_{i}$ divides its weight $w_{i}$ into two equal positive real parts $x, y$ so that $w_{i}=x+y$.

2) $\quad P_{i}$ sends a basic message $B(x)$ along with a weight $x$.

3) $\quad P_{i}$ updates its current weight as $w_{i}=y$.

4) On reception of the basic message, $P_{j}$ 's weight increases as $w_{j}=w_{j}+x$.

Any process in the system can become passive at any time. When a process other than the leader becomes passive, it sends a control message to the leader for submitting its weight. After sending the control message this process sets its weight to 0 . Any passive process can become active again by receiving a basic message from some other process. The following are the invariants in the protocol:

- $\quad$ Each process and in-transit messages in the system have a non-zero weight at any time.

- If we take the sum of the weights of all processes and in-transit messages at any time, it is always 1 .

When the leader becomes passive, it accumulates all the weights in the system. If accumulated weight becomes equal to 1 , the leader calls termination. Weights should be handled precisely. Fractional values of all weights make it nearly impossible to make the sum to 1 again due to rounding errors of float values. This problem can be solved by representing the weight using two integer values as $[1, n]$ instead of $1 / n$.

\section{A. Flow-detecting Scheme for Flushing/Freezing of Channels}

In case of faulty distributed system, some processes may fail during a computation. Overall weight of all processes can be less than 1 due to holding weights of failed processes and weights carried by undelivered messages in the channels. This problem is solved by introducing a flow detecting scheme. Let $H$ be a subsystem which contains all healthy processes and all their communication channels. During a computation, the weight change in $H$ at time interval $I$ is equal to the difference of weight flowing into $H$ and the weight flowing out of $H$. With the help of this scheme, the weight information of failed processes can be obtained from the outgoing weight records of healthy processes in the system because each process keeps the record of incoming and outgoing weights.

Assume that the intended system provides the facility of flushing or freezing of channels connected to faulty processes. Flushing or freezing mean preventing and ceasing further communication between a healthy and a faulty process. There is no global clock in the system that makes it very difficult to get global views of the system weight. A snapshot is taken to get the global views. The leader sends the snapshot request to all the healthy processes. On reception of this request, each healthy process flushes or freezes all of its communication channels connected to faulty processes and submits its incoming and outgoing weights to the leader. The leader uses these incoming and outgoing weight values to calculate the overall weight of the system.

\section{B. Data Structure of Weight-throwing Protocol}

Before the formal modeling of the protocol we need to know the specific keywords and data structure used in the protocol. Let $P_{i}$ be any process in the system where $i=1 \ldots n$, $n$ is any arbitrary positive number. The data structure for $P_{i}$ is given in Table I.

\section{MODELING IN UPPAAL}

Our formal specification in UPPAAL has three participants, i.e. the Termination, the MessageBuffer and the SnapBuffer. The main process is the Termination process. This process receives and sends messages to other processes to communicate. Each message holds some non-zero weight. A process adds the incoming weight to its current weight when it receives a message. The communication is asynchronous. The MessageBuffer process holds the basic and control messages when receiver is not ready to receive them. These messages are moved to their receivers when they become ready. The SnapBuffer process temporarily stores the snapshot request messages sent by the current leader. It then sends the snapshot request messages to all the healthy processes to inform them the faulty set of processes known to the leader. This process also temporarily keeps the snapshot reply messages sent by the processes to the leader. These messages are delivered to the leader when it becomes ready.

The protocol has two parts. In first part, the termination detection is done using a fault-free distributed system. In the second part, a faulty distributed system is used to detect proper termination of processes. In faulty distributed system any number of processes may tend to fail. We present the formal specification of both parts separately to check the correctness of the protocol in both cases. 
TABLE I. INFORMAL SPECIFICATION OF WEIGHT-THROWING PROTOCOL

\begin{tabular}{|c|c|}
\hline leader $_{i}$ & $\begin{array}{l}\text { This variable stores id of current leader. At the } \\
\text { beginning, leader }{ }_{i}=1 .\end{array}$ \\
\hline$w_{i}$ & $\begin{array}{l}\text { This field keeps the value of weight for each process } \\
P_{i} \text {. Initially, the value of } w_{i} \text { is } 1 / n \text {, where } n \text { is the } \\
\text { number of processes. }\end{array}$ \\
\hline sum $_{i}$ & $\begin{array}{l}\text { This variable records total weight in the system as- } \\
\text { sumed by the leader. It is a real number. At the } \\
\text { beginning, sum } m_{i}=1 \text {. }\end{array}$ \\
\hline$I N_{i}[1 \ldots n]$ & $\begin{array}{l}\text { This is an array of real numbers. The } I N_{i}[j] \text { keeps } \\
\text { the record of all weights thrown out from process } \\
P_{j} \text { to } P_{i} \text {. At the beginning, } I N_{i}[j]=0 \text { for all } \\
j=1 \ldots n \text {. }\end{array}$ \\
\hline$O U T_{i}[1 \ldots n]$ & $\begin{array}{l}\text { This is an array of real numbers. The } O U T_{i}[j] \text { stores } \\
\text { all the weights thrown to process } P_{j} \text { from } P_{i} \text {. At the } \\
\text { beginning, } O U T_{i}[j]=0 \text { for all } j=1 \ldots n \text {. }\end{array}$ \\
\hline$F_{i}$ & $\begin{array}{l}\text { This array represents a set of faulty processes. If a } \\
\text { process } P_{i} \text { knows } P_{j} \text { to be faulty and } P_{i} \text { has flushed } \\
\text { or frozen all the channels to } P_{i} \text { then it belongs to } F_{i} \\
\text {. At the start } F_{i}=\phi \text {. }\end{array}$ \\
\hline$S N_{i}$ & $\begin{array}{l}\text { This array contains set of all processes to which } \\
\text { snapshot request is to be sent. Let's suppose } P_{i} \\
\text { initiates the snapshot request. If a process } P_{j} \text { replies } \\
\text { to the request or it is found faulty by } P_{i} \text { then it } \\
\text { is removed from the } S N_{i} \text {. Second snapshot can be } \\
\text { initiated only when } S N_{i} \text { becomes empty. At the start } \\
S N_{i}=\phi \text {. }\end{array}$ \\
\hline temp_sum ${ }_{i}$ & $\begin{array}{l}\text { This field stores a real number. During the snapshot, } \\
\text { it temporarily calculates the total remaining weight. }\end{array}$ \\
\hline consistent $_{i}$ & $\begin{array}{l}\text { This field indicates a boolean value which keeps the } \\
\text { record of a snapshot's consistency. }\end{array}$ \\
\hline$B(x)$ & This represents the basic message with a weight $x$. \\
\hline$C(x)$ & $\begin{array}{l}\text { This indicates a control message. The control message } \\
\text { is used for reporting the weight } x \text { to the leader of the } \\
\text { system. }\end{array}$ \\
\hline Request $\left(F_{i}\right)$ & $\begin{array}{l}\text { This represents the message for snapshot request that } \\
\text { is sent by the current leader of the system } P_{i} \text {. With } \\
\text { the help of } F_{i} \text {, message receiving process is informed } \\
\text { about the set of faulty processes already known to the } \\
\text { leader. }\end{array}$ \\
\hline $\operatorname{Reply}\left(F_{i}, I N_{i}, O U T_{i}\right)$ & $\begin{array}{l}\text { This indicates the reply to the leader's request mes- } \\
\text { sage. This reports the state of the replying process. }\end{array}$ \\
\hline
\end{tabular}

\section{Model 1: Termination Detection in A FAULT-FreE Distributed SySTEM}

We specify all the processes of fault-free part of the protocol. The Termination and the MessageBuffer are the participants in Model1. We present the functionality and formal specification of these participants in this section.

\section{A. Channels}

This protocol uses four channels which are described below. To model the functionality of termination detection in a fault-free distributed system, we use hand shaking channels. The working of these channels is described below:

1) basicMessageS: This channel is very important because the system uses this channel for the basic message communications. It sends basic messages to the MessageBuffer to hold them until their receivers become ready.

2) basicMessageR: For moving stored basic messages from the MessageBuffer to the receiver process, system uses basicMessageR channel.

3) controlMessageS: This is a channel for control message communications. System uses this channel to send control messages to the MessageBuffer to hold them until the leader becomes ready.
4) controlMessageR: This channel moves stored control messages from MessageBuffer to the leader.

\section{B. Global Declarations}

Some variables and arrays are declared globally so that each participant can access them and use them according to their needs. Table II represents the global declarations and data types for Model1.

\section{TABLE II. Global Declarations for Model1}

\begin{tabular}{|l|l|}
\hline numberofweights & $\begin{array}{l}\text { This is a constant that represents the total parts of } \\
\text { weight. }\end{array}$ \\
\hline max_weight_limit & $\begin{array}{l}\text { This describes the maximum value of weight that can } \\
\text { be sent through communication channels. }\end{array}$ \\
\hline maxproc & $\begin{array}{l}\text { It is a variable that tells the number of concurrent } \\
\text { instances of Termination process. }\end{array}$ \\
\hline max_proc_id & $\begin{array}{l}\text { This variable stores the highest id of concurrent in- } \\
\text { stances of the Termination process. }\end{array}$ \\
\hline leader & $\begin{array}{l}\text { This variable keeps id of the current leader of the } \\
\text { system. }\end{array}$ \\
\hline W1 and W2 & $\begin{array}{l}\text { These variables record the weights when a basic or a } \\
\text { control message is received. }\end{array}$ \\
\hline Out_arr[] and In_arr[] & $\begin{array}{l}\text { Two customized data structures Weight_out and } \\
\text { Weight_in are introduced to define the Out_arr[] and } \\
\text { In_arr[] arrays respectively. The Out_arr[] keeps the } \\
\text { records of out going weights and In_arr[] stores the } \\
\text { records of incoming weights of each process of the } \\
\text { Termination process. }\end{array}$ \\
\hline
\end{tabular}

\section{The Automaton for Termination Process in Model1}

The automaton for the Termination process is depicted in Fig. 2. The protocol model has a number of parallel processes, each of which is triggered by a certain communication among each other. The specification of the Termination process comprises four communicative choices i.e. sending basic message, receiving basic message, sending control message and receiving control message.

For overall working of the Termination process, we discuss the functionalities which take place between active and $A l$ states. The basicMessageS! sends two weight values of basic message to the MessageBuffer for any other process. Two weights actually represent the single weight because we are using two values (numerator and denominator) just to avoid floating point errors. The guards for weight values, limit the number of basic messages that a process can send to other processes to reduce transition state space. Going from $A l$ to active state, the updateOut() function updates the Out_arr[] to record the outgoing weights. Also the weight[1] value is doubled because doubling the denominator value, overall weight of a process becomes half. For a process taking a transition from the active to A2 state, the channel basicMessageR? receives two weight values and sender id from its MessageBuffer to record incoming weight against a specific sender. In next transition, the updateIn() function updates the In_arr [] to record incoming weight from that specific process. The updateWeight() function records the overall current weight of receiver process. Same procedure is followed when going from passive to active state because both the transitions are identical and perform exactly same functionality. While taking transitions from active to $A 3$ and $A 3$ to passive state, the channel controlMessageS! sends weight values of this process alongwith its id to the leader. The function updateIn() 
receiver:int[0, max_proc id],

second id:int $[0$, max proc id $]$

basicMessageS[pid][weight[0]][weight[1] *2][receiver][second_id]! basicMessageR[sender][wei1][wei2][pid]?

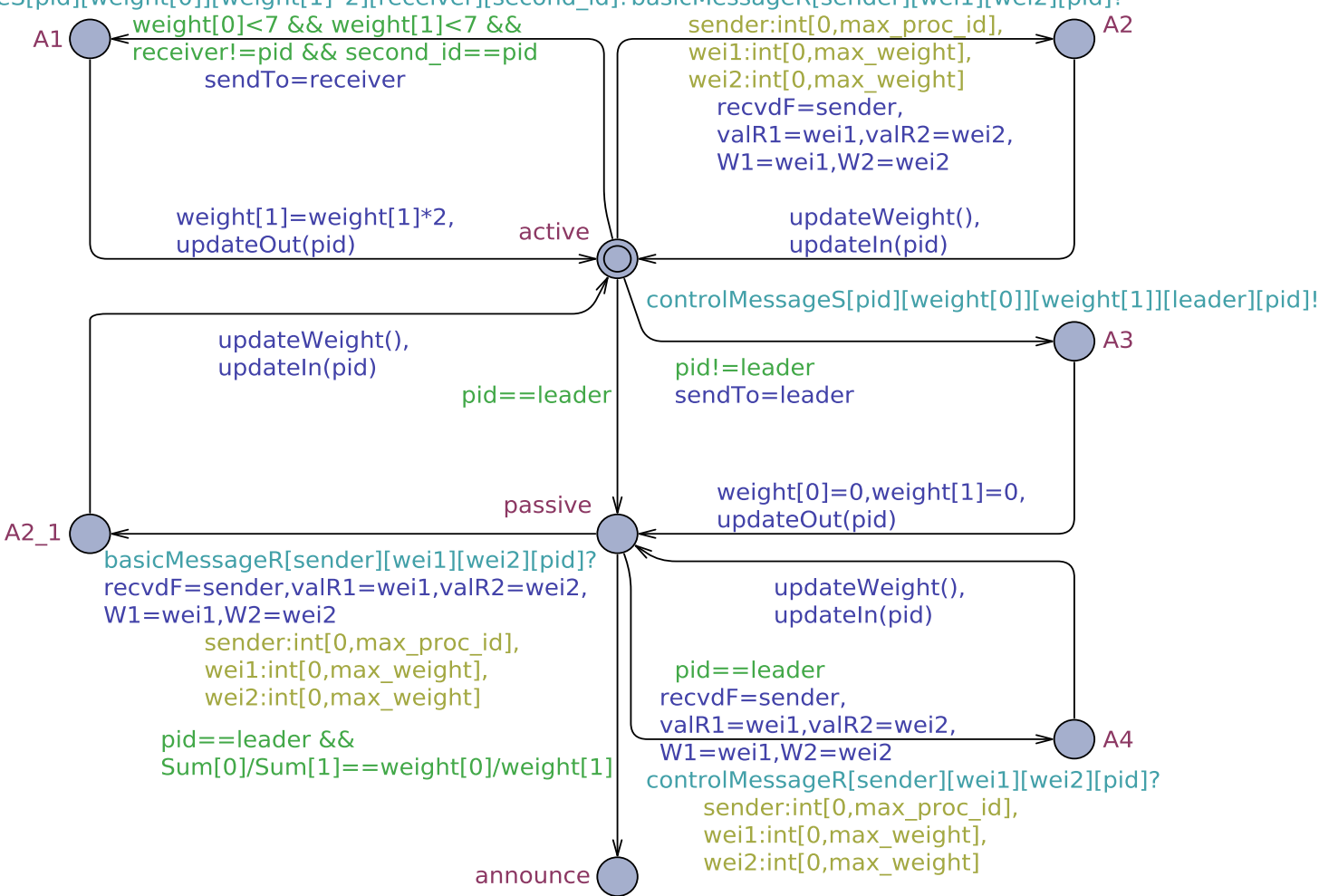

Fig. 2. Formal model for Termination process in Model1.

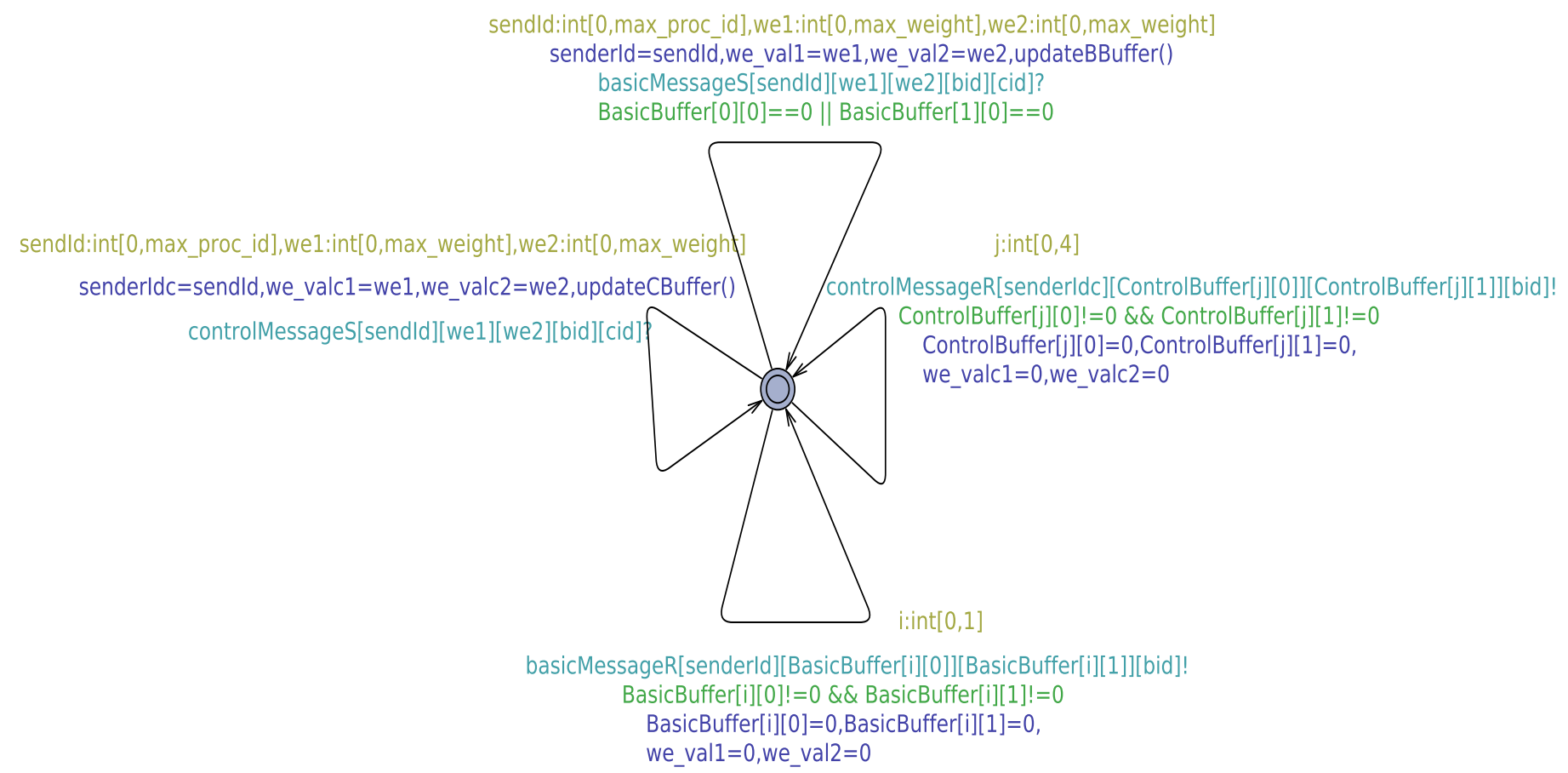

Fig. 3. Formal model for MessageBuffer process. 
updates Out_arr[] of this process. The value of weight[0] and weight $[\overline{1}]$ is set to zero to make sure that all the weight is transferred to the leader. The working of transitions from passive to A4 is similar to moving from active to A2 state. The only difference is, in controlMessageR? channel, the leader receives the control message from MessageBuffer. All other variable definitions are exactly same. Taking transition from A4 to passive state uses exactly same functions as described previously for $A 2$ to active state. Only the leader can take the transition from passive to announce state. The leader takes this transition only when it has collected all the weight from all the processes through control messages. If this weight becomes equal to the weight defined at the beginning of the computation, the leader announces termination.

The local declaration and data types of Termination process are given in Table III.

TABLE III. LOCAL DECLARATIONS FOR Termination PROCESS IN MODEL1

\begin{tabular}{|l|l|}
\hline valRI and valR2 & $\begin{array}{l}\text { These variables temporarily store first and second value of } \\
\text { weight respectively at different transitions. }\end{array}$ \\
\hline recvdF and sendTo & $\begin{array}{l}\text { System uses recvdF variable to store process id of the } \\
\text { message sender and sendTo records process id of the } \\
\text { message receiver. }\end{array}$ \\
\hline weight [] & $\begin{array}{l}\text { weight array permanently stores the two weight values } \\
\text { for every process at any time. }\end{array}$ \\
\hline Sum [] & $\begin{array}{l}\text { The Sum[] a value container that represents the total } \\
\text { weight of the system that is actually 1. }\end{array}$ \\
\hline tempIn[] & $\begin{array}{l}\text { This array temporarily stores the sum of all incoming } \\
\text { weights to a process. }\end{array}$ \\
\hline tempOut[] & $\begin{array}{l}\text { tempOut[] keeps the sum of all the outgoing weights } \\
\text { from a process. }\end{array}$ \\
\hline
\end{tabular}

In UPPAAL system models, we can declare functions with in the process or alongwith global declarations. The functions can have return types and passing parameters. The Termination process also uses some functions to perform its functionality.

1. updateWeight(): This function is very important because it is called at different transitions when a basic or a control message is received. If the receiver has zero weight, the received weight is directly moved to weight[] of the process. If the process already has some non-zero weight then function adds the incoming and current weight to calculate the new weight.

2. updateIn(): A process uses this function when it receives a control or a basic message. This function updates the In_arr[] to record the incoming weight from a particular process. The incoming weight is directly moved to the In_arr[] if receiver is receiving the first message from the sender. The current weight and incoming weight is added to update the incoming weight in In_arr [] if the process already has received some messages from the same sender.

3. updateOut(): A process calls this function when it sends a control or basic message. This function updates the Out_arr [] to record the outgoing weight to a particular process. The outgoing weight is directly moved to the Out_arrarray if receiver is receiving the first message from the sender. The current weight and outgoing weight is added to update the Out_arr [] if the process already has sent some messages to the same receiver.

\section{The Automaton for MessageBuffer Process}

The automaton for the MessageBuffer process is depicted in Fig. 3. The process has three instances. The specification of the MessageBuffer process in the protocol provides four communication choices i.e. receiving a basic message from the Termination process, sending a stored basic message to the Termination process, receiving a control message from the Termination process and sending a stored control message to the Termination process.

Now we discuss the functionalities for the MessageBuffer process when a basic message is received through basicMessageS? channel. The function updateBBuffer() updates the basic message buffer to keep the record of this incoming basic message until it is not delivered to the respective recipient. The basicMessageR! channel sends the stored basic message when the receiver Termination process becomes ready. The two guards prevent the communication between MessageBuffer and Termination process when their is no stored message. The function updateCBuffer() is called to update the control message buffer to keep the record of this incoming control message until its recipient is not ready. This happens when a control message is received through controlMessageS? channel. The controlMessageR! sends the stored control message when the leader becomes ready. The two guards prevent the communication between the MessageBuffer and the leader when their is no stored control message for the leader.

The local declaration and data types of the MessageBuffer process are given in Table IV.

\section{TABLE IV. LOCAl DeClarations For MessageBuffer Process}

\begin{tabular}{|c|c|}
\hline we_vall and we_val2 & $\begin{array}{l}\text { The variables store first and second value of } \\
\text { weight[] for receiving and sending basic mes- } \\
\text { sages at different transitions of MessageBuffer } \\
\text { process. }\end{array}$ \\
\hline senderId and senderIdc & $\begin{array}{l}\text { senderld variable stores the process id of the } \\
\text { basic message sender process and senderIdc } \\
\text { keeps the process id of the control message } \\
\text { sender process. }\end{array}$ \\
\hline we_valcl and we_valc2 & $\begin{array}{l}\text { These variables contain the first and second } \\
\text { value of weight[] for receiving and sending } \\
\text { control messages at different transitions of } \\
\text { MessageBuffer. }\end{array}$ \\
\hline BasicBuffer[] and ControlBuffer[] & $\begin{array}{l}\text { The BasicBuffer array list is to store the two } \\
\text { basic messages for each process at any time. } \\
\text { The limit to store only two basic messages is } \\
\text { for achieving reduced transition state space. } \\
\text { The ControlBuffer[] can store five control } \\
\text { messages for the leader process at any time. }\end{array}$ \\
\hline
\end{tabular}

The MessageBuffer process uses some functions to perform different tasks on different transitions. We discuss these functions below.

1. updateBBuffer(): It is called on a transition when a basic message is received. It locates the available free space in BasicBuffer[] and then stores the incoming basic message at that space.

2. updateCBuffer(): A transition calls this function when it receives a control message at MessageBuffer process. The function checks the free space in ControlBuffer [] and then stores the incoming control message at that position. 


\section{Model2: Termination Detection in a Faulty DISTRIBUTED SYSTEM}

We specify all the concurrent processes of faulty part of the protocol. The three participants are the Termination, the MessageBuffer and the SnapBuffer. The functionality and formal specification of these participants is presented in this section. The functionality of the MessageBuffer process is already described in Model1. Here we discuss the functionality of remaining participants.

\section{A. Channels}

This protocol uses nine channels for communication among processes. Four of them are same as described in Model1. The other five channels are described here in detail.

1) failReport: The Termination process uses this channel when a process fails. It tells the failed status of a process to other processes. On the other side, a process receives the status of a failed process using this channel.

2) failRequest: This channel sends snapshot request message to the SnapBuffer process.

3) failRequestR: The channel sends the stored snapshot request message from SnapBuffer process to the recipient Termination process.

4) failReply: This channel is used to send the snapshot reply message to the SnapBuffer process.

5) failReplyR: The channel delivers the stored snapshot reply message from SnapBuffer process to the recipient Termination process.

\section{B. Global Declarations}

This system is modeled for termination detection in faulty distributed environment. This is the enhancement of the Model1 (fault-free model). Therefore some global variables are common in both the systems. We present here the description of variables that are not present in Modell but are present in Model2. The global declarations for Model2 are described in Table V.

\section{TABLE V. Global Declarations for Model2}

\begin{tabular}{|l|l|}
\hline FIn[] and FOut[] & $\begin{array}{l}\text { The FIn array stores all the incoming weights and FOut[] } \\
\text { saves the outgoing weights of failed processes which are } \\
\text { known to the leader during the snapshot. }\end{array}$ \\
\hline FI_FO_Diff[] & $\begin{array}{l}\text { The FI_FO_Diff array stores the difference of all the incoming } \\
\text { and outgoing weights of failed processes known to the leader } \\
\text { during the snapshot. }\end{array}$ \\
\hline S[] & $\begin{array}{l}\text { System uses } S \text { array to store ids of all instances of Termination } \\
\text { process participating in the system. }\end{array}$ \\
\hline F[] & $\begin{array}{l}\text { The } F \text { is a global array. It records the failed processes known } \\
\text { to each process. The value may be different for each process. }\end{array}$ \\
\hline Ftemp[] & $\begin{array}{l}\text { Ftemp[] keeps the actual record of failed processes in the } \\
\text { system. }\end{array}$ \\
\hline
\end{tabular}

\section{The Automaton for Termination Process in Model 2}

We have discussed the Termination process for Model1 in previous section. The automaton for Termination process in Model 2 is depicted in Fig. 4. The specification of Termination process has ten communicative choices four of which are same as in Model1. The other six choices are sending snapshot request message, receiving snapshot request message, sending snapshot reply message, receiving snapshot reply message, sending fail report message and receiving fail report message.

Now Termination has nine actions from $A 1$ to $A 5$ and from $F 1$ to $F 4$. The actions $A 1$ to $A 5$ are already discussed in Model1. So we discuss here only the actions $F 1$ to F4. Fig. 5 represents the formal model for F1. The failReport? channel detects the failed process when no snapshot is in progress. In next transition, the process adds the failed process in its $F[]$ and Flush[]. The function Leader() is called to determine the leader. If current process is not the leader then it reaches to active state. If this process is the leader then it reaches to Snap state and starts calculating the healthy processes to send them snapshot request message. The SnapBuffer stores this message until the receiver of this message is not ready. After sending these messages the process is allowed to reach at active state.

Fig. 8 represents the formal model for F2. A process receives the stored snapshot request message from SnapBuffer process through failRequestR? channel. Then it sends the snapshot reply message to the SnapBuffer for the leader through failReply! channel. It records the new leader. It matches and updates its $F[]$ to record the failed processes known to the leader.

Fig. 6 shows the formal model for F3. The leader receives the stored snapshot reply message from SnapBuffer process through failReplyR? channel. It checks the difference of $F[]$ of sender and its own $F[]$. The snapshot is marked as inconsistent if this difference is greater than zero or the snapshot is not consistent. The process which has sent this snapshot reply message is removed from $S N[]$. The process reaches to active state if a snapshot is in progress otherwise reaches to Snap state.

Fig. 7 represents the formal model for F4. The failReport? channel detects a failed process. The action makes sure that a snapshot is already in progress. In next transition this process adds the failed process in F[] and Flush []. It also sets the snapshot as inconsistent. Then after removing the failed process from $S N[]$, if still $S N[]$ is non empty then process calls the snapshot.

Fig. 9 represents the formal model for the process when it fails. At failure, the process updates the FTemp[] to record its entry in that array and moves from active to fail state. The channel failReport! at fail state continuously tells other processes that its status is failed.

We have discussed some local declarations of Termination process in Modell. Now we discuss the local declarations of remaining variables in Table VI.

calSN(), calcDiff(), isAvailable(): The three functions perform the combined functionality of identifying the healthy processes for sending snapshot request messages. The function calSN() calls the calcDiff() function. The calcDiff() function calls isAvailable() function for every process id. If a true value is returned it means that the process is present in the $F[]$ of calling process and there is no need to send snapshot request message to that process.

$\operatorname{Add} \operatorname{In}()$ : The function $\operatorname{Add} \operatorname{In}()$ calculates the total incoming weights of all the failed processes known to the current leader during the snapshot. It adds the incoming weights of every failed process to make a sum of incoming weights in FIn[]. 


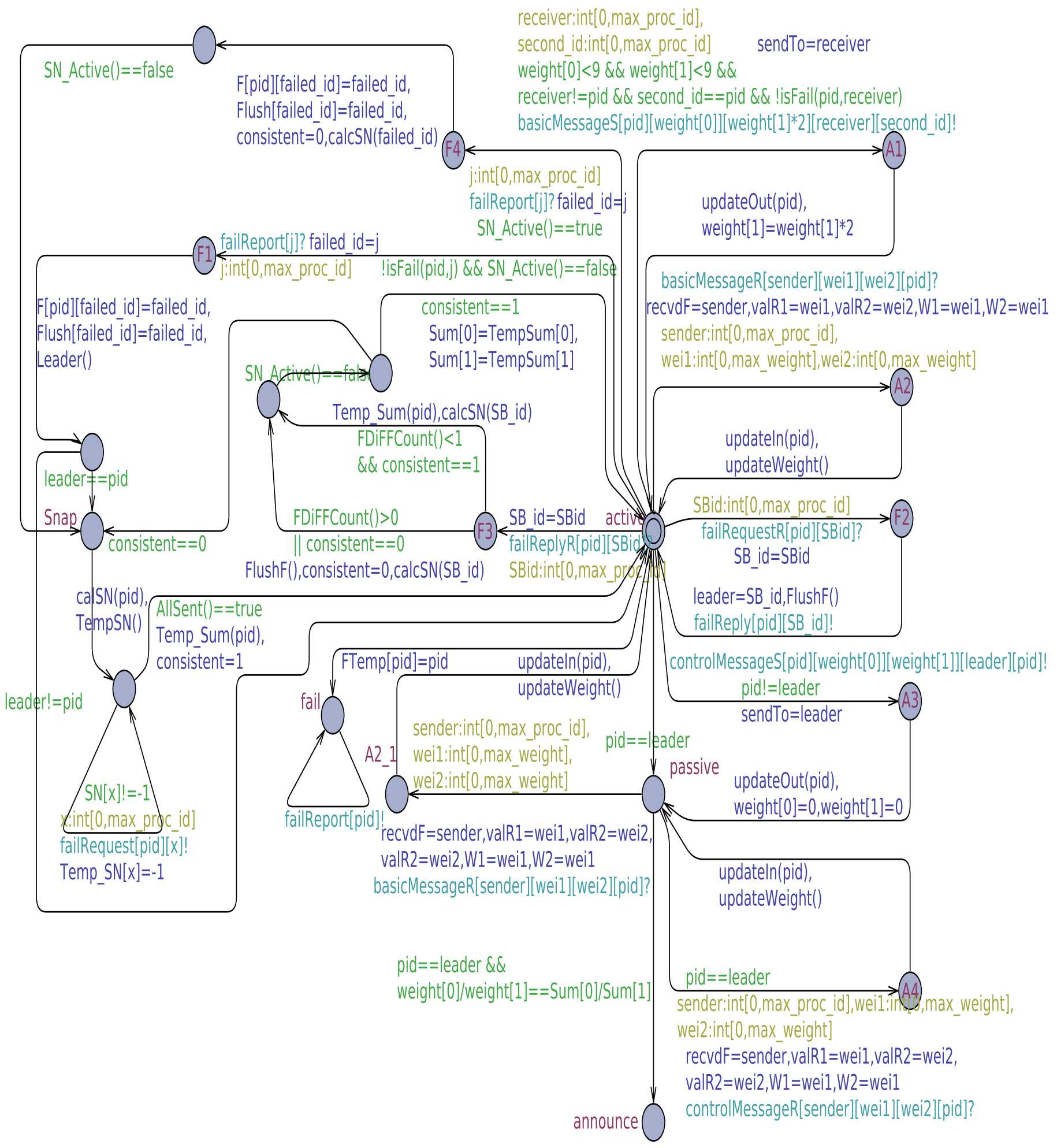

Fig. 4. Formal model for Termination process in Model2. 


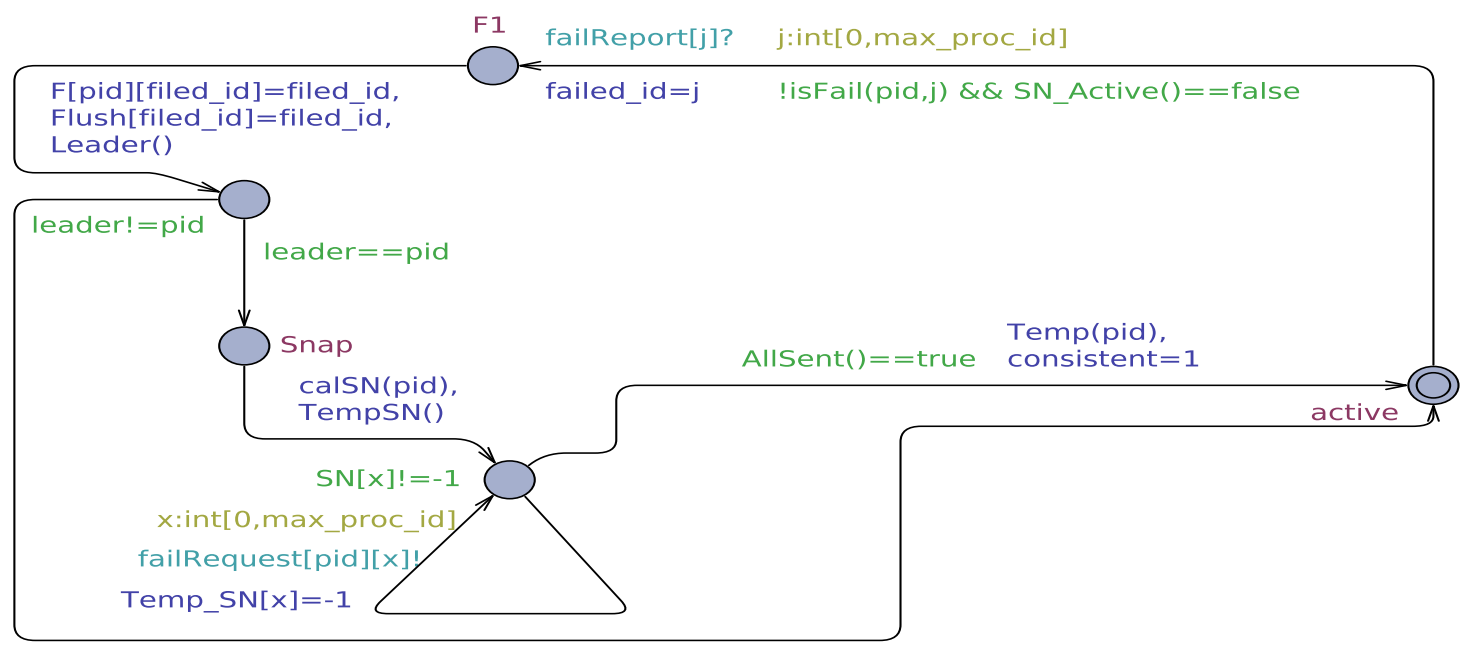

Fig. 5. Formal model for Action F1.

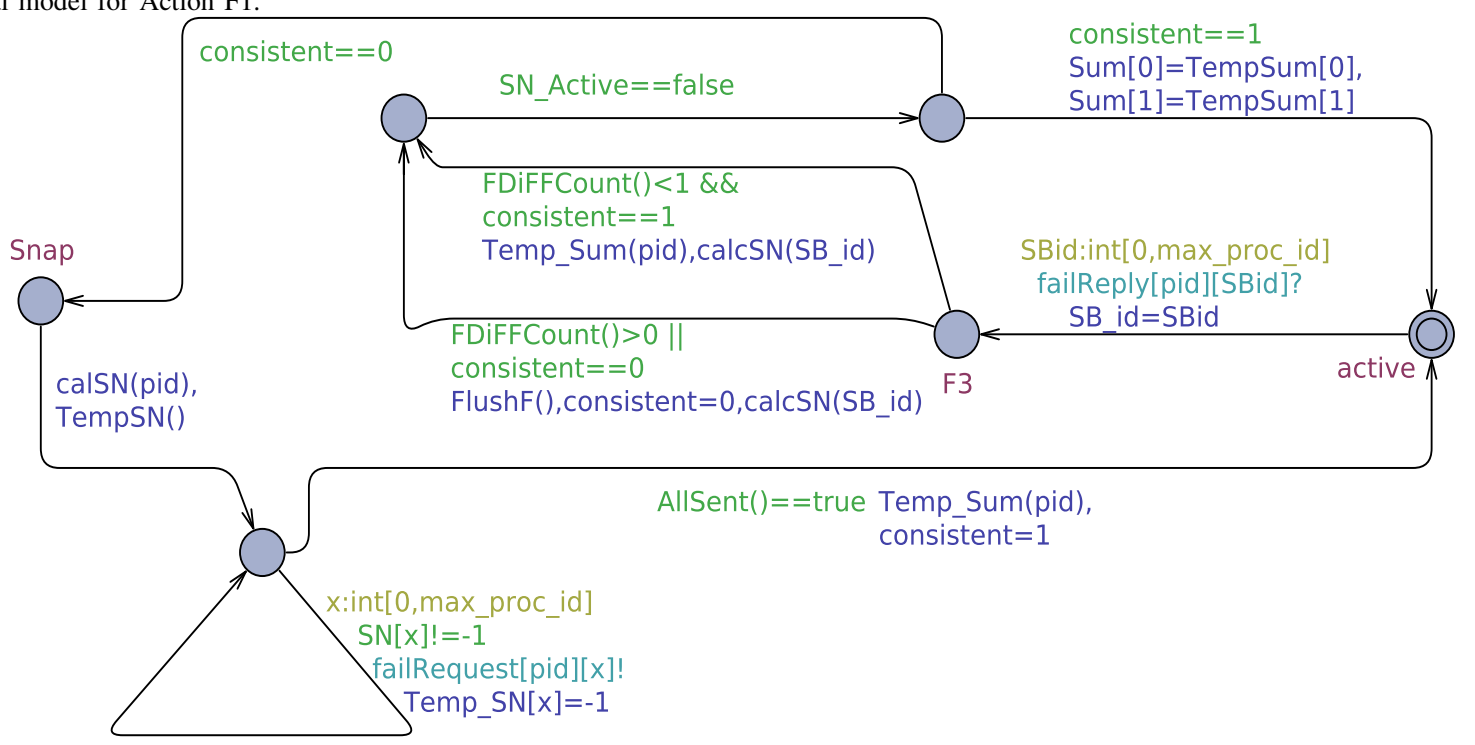

Fig. 6. Formal model for Action F3.

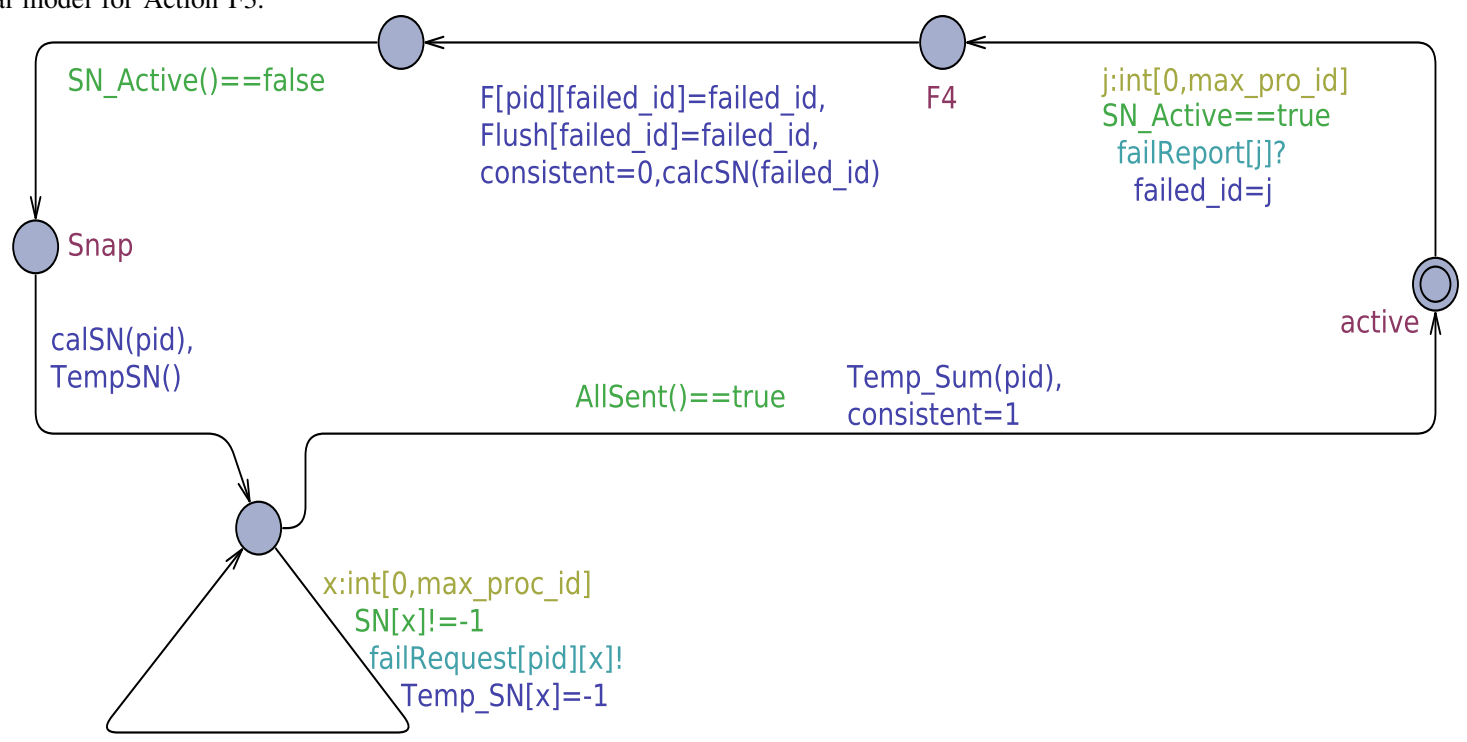

Fig. 7. Formal model for Action F4. 


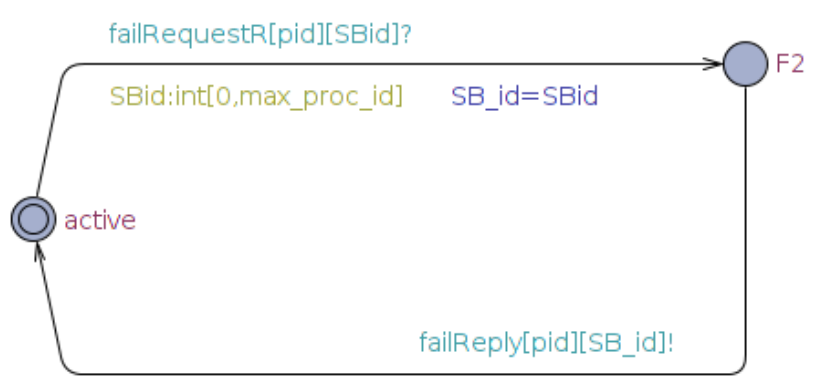

leader $=\mathrm{SB}_{-}$id, $\mathrm{FlushF}()$

Fig. 8. Formal model for Action F2.

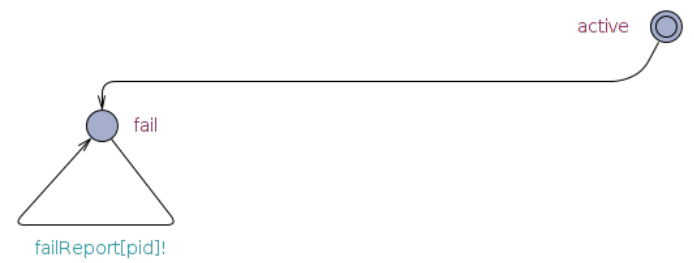

Fig. 9. Formal model for failed process.

TABLE VI. LOCAL DEClaRATIONS FOR TERMINATION PROCESS IN MODEL2

\begin{tabular}{|l|l|}
\hline leader & $\begin{array}{l}\text { This variable stores the id of the current leader of the system } \\
\text { known to a process. It may be different for each process. } \\
\text { These variables record the ids of the message sender process } \\
\text { at different transitions. }\end{array}$ \\
\hline consistent $S B \_i d 2$ & $\begin{array}{l}\text { The boolean variable that shows the consistency of the } \\
\text { snapshot request } \text { sent by the leader. }\end{array}$ \\
\hline TempSum[] & $\begin{array}{l}\text { The TempSum array stores the sum of all the weights during } \\
\text { the snapshot. }\end{array}$ \\
\hline SN[] & $\begin{array}{l}\text { The } S N[] \text { keeps the set of processes to which the snapshot } \\
\text { request is to be sent by the leader. }\end{array}$ \\
\hline Temp_SN[maxproc] & $\begin{array}{l}\text { The Temp_SN array temporarily records the set of processes } \\
\text { to which the snapshot request message is to be sent by the } \\
\text { leader. After sending the snapshot request to a process, the } \\
\text { sender removes this process from Temp_SN[]. This array } \\
\text { becomes empty after sending snapshot request } \text { message to } \\
\text { all the healthy processes. }\end{array}$ \\
\hline failed_id & $\begin{array}{l}\text { A local variable that stores the id of the failed process at } \\
\text { different transitions. }\end{array}$ \\
\hline Flush[] & $\begin{array}{l}\text { This array keeps the record of failed processes for which all } \\
\text { further communications are flushed. }\end{array}$ \\
\hline Stemp[] & $\begin{array}{l}\text { The Stemp array contains the list of all the instances of } \\
\text { Termination process taking part in the system. }\end{array}$ \\
\hline
\end{tabular}

AddOut(): It calculates the total outgoing weights of all the failed processes known to the current leader during the snapshot. It then adds the outgoing weights of every failed process to make a sum of outgoing weights in FOut[].

FIin_FOut_Diff(): This function checks the difference of incoming weights and outgoing weights. It uses the FIn[] for incoming weights and FOut[] for outgoing weights. It subtracts the outgoing weights from incoming weights. Then it moves the difference to FI_FO_Diff[].
Temp_Sum(): This function adds the FI_FO_Diff values with $[1 / n]$. The sum is stored in TempSum[]. The Temp_Sum() function calls FIn_FOut_Diff() function, the FIn_FOut_Diff() function calls $A d d$ Out() function, the AddOut() function calls AddIn() function. In this way all the calculations are done properly. The benefit of calling functions inside other functions is that we just call the Temp_Sum() function on a transition and all the calculations for TempSum[] are done properly.

AllSent(): It checks if the snapshot requests have been sent to all the healthy processes.

isFail(): A process uses this function to check the entry of a failed process in $F[]$. If record found then current process can not detect the failure of this process again.

Leader(): The Leader() function makes the new leader when a process detects failure of some other process. This function is very important because if the leader fails then the system needs a new leader to collect the weights and send snapshot request messages. This function makes the leader to a healthy process with minimum id. If the failing process is not the leader then this function again selects the previous leader.

$S N \_$Active(): System uses this function at different transitions to check if a snapshot is already in progress. The function returns a true value if snapshot is already in progress otherwise returns a false value.

FDiFFCount(): It calculates the difference of F[] of snapshot reply sending process and the $F[]$ of the leader when the leader receives the snapshot reply message.

\section{The Automaton for SnapBuffer Process}

The automaton for the SnapBuffer process is depicted in Fig. 10. The process is initiated four times to make four instances, each of which is triggered by a certain communication with the Termination process. The specification of the SnapBuffer process in the protocol has following communicative choices:

1) Receiving a snapshot request message from leader to store it.

2) Sending a stored snapshot request message to a Termination process.

3) Receiving a snapshot reply message from a Termination process to store it.

4) Sending a stored snapshot reply message to leader.

The process receives a snapshot request message through failRequest? channel. The guard makes sure that buffer is empty and can receive this message. After receiving this message the IsEmpty variable is assigned a false value to show that now buffer is non-empty. The SnapBuffer process delivers the stored snapshot request message to the recipient through failRequestR! channel. The guard makes sure that buffer contains a message for sending. After sending this message the variable IsEmpty is set true to show that now buffer is empty again. The SnapBuffer process receives a snapshot reply message through failReply? channel. The guard ensures that buffer is empty and can receive this message. After receiving this message the IsEmpty 2 variable is assigned a false value to show that now buffer is non-empty. The SnapBuffer process sends the stored snapshot reply message to the leader 


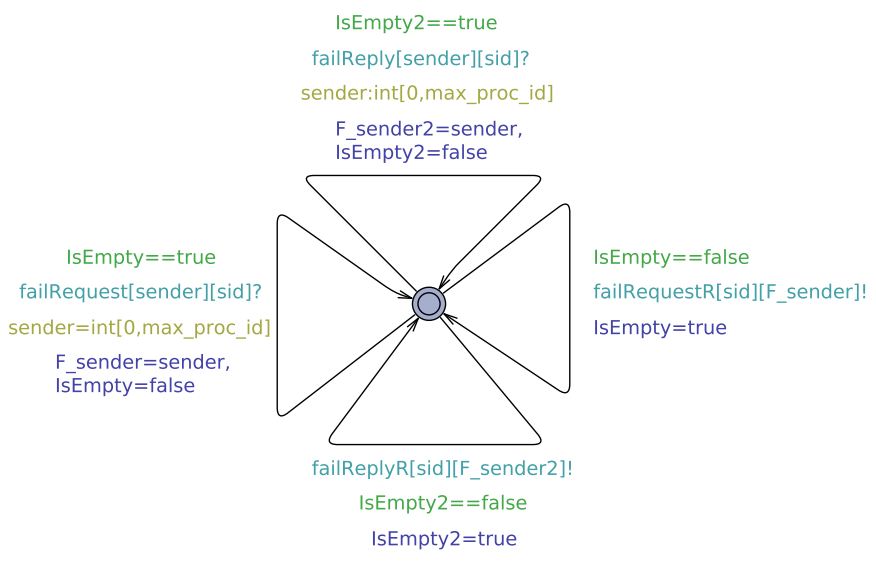

Fig. 10. Formal model for SnapBuffer process.

through failReplyR! channel. After sending the message the variable IsEmpty2 is set true to show that now buffer is empty again.

The local declarations of SnapBuffer process are described in Table VII.

TABLE VII. Local DeClarations For SnapBuffer Process

\begin{tabular}{|l|l|}
\hline$F_{-}$sender and $F_{-}$sender2 2 & $\begin{array}{l}\text { These variables store the process id of the snapshot } \\
\text { request sender and process id of the snapshot reply } \\
\text { sender, respectively. }\end{array}$ \\
\hline IsEmpty and IsEmpty2 & $\begin{array}{l}\text { Boolean variables which indicate free space for incom- } \\
\text { ing snapshot request message and incoming snapshot } \\
\text { reply message, respectively. }\end{array}$ \\
\hline
\end{tabular}

\section{FunCtional ReQuirements}

The functional requirements illustrate the behaviour of the system and explain what an intended system should do. In other words, they describe the functionality of the system. Every protocol has some functional requirements. We discuss and verify these requirements for both models separately.

\section{A. Functional Requirements for Model1}

We extract three functional requirements from the protocol for Model1. These are given as:

R1: No deadlock is supposed to be there except when the leader process is at announce state and all other processes are at passive state. This indicates that all the processes are idle and the leader has collected all the weights successfully resulting in proper termination of the system.

R2: This requirement states that after doing certain communications and collecting the weights of other processes, the leader process eventually reaches at announce state.

R3: According to this requirement, after doing certain message communications all the processes must be idle at passive state. All the processes eventually reach at passive state.
There are three invariants given in the protocol at page 12 of [7]. These invariants are expected to be preserved by the system.

INV1: In Model1, no process fails. It means all the processes are healthy. The process with minimum id is the leader of the system. This invariant is for all the healthy processes other than the leader. This invariant states that at any time, if a process is at passive state then it must have a zero weight. Similarly, if a process has zero weight then it must be at passive state.

INV2: This invariant is related to message sending. All processes can pass basic messages to each other. Every process can also send control messages to the leader. A process sends some weight with $b a$ sic and control messages. This invariant describes that the weight sent with any message must be greater than zero.

INV3: At the start of the computation, every process is given an equal initial weight. A process sends some of its weight when it sends a basic or control message. Each process receives some weight when it receives that message. It updates its weight after sending or receiving a message. All processes also record their incoming and outgoing weights. This invariant states that for all healthy processes at any time, the sum of current weight and all outgoing weights of a process must be equal to the sum of its initial weight and all incoming weights.

\section{B. Functional Requirements for Model2}

For Model2, we extract three functional requirements.

R1: This requirement describes that some process reaches at the announce state to make sure that all the weights are collected and the system is terminated properly.

R2: A faulty process cannot be the leader of the system. This requirement is not satisfied. We discuss a scenario in which this requirement is violated. We have 4 instances of the Termination process. These are $p 0, p 1, p 2$ and $p 3$. The $p 0$ is the leader of the system. The leader sends the snapshot request message to all the processes. These messages are yet stored in buffers and not delivered to the recipients. Meanwhile, the leader fails. The $p l$ detects the leader to be faulty and calculates the new leader with minimum id from healthy processes. It becomes the leader itself. But in future, when it receives the stored snapshot request message sent by $p 0$, it makes the $p 0$ as the leader of the system. The $p 0$ is faulty and is supposed to be the leader of the system again. That is why this is the clear violation to this functional requirement.

R3: This requirement states that every time the leader fails, the snapshot is called. The healthy process with minimum id calls the snapshot. But this requirement is trivially violated when the $p 0$ fails and the $p l$ becomes passive without detecting the 
fault. The $p 2$ and $p 3$ also become passive. Now the snapshot is never called by any process.

The three invariants discussed for Modell must be preserved for Model2 also.

\section{Formal SPECIFICATION OF REQUIREMENTS}

In this section, we describe formal specification of the requirements and invariants. We also present the formalism for these requirements.

\section{A. Requirement Formal Specification for Modell}

According to requirement R1, there should not be a deadlock if the leader is not at announce state or any of the other process is not at passive state. The formula for this requirement is given as.

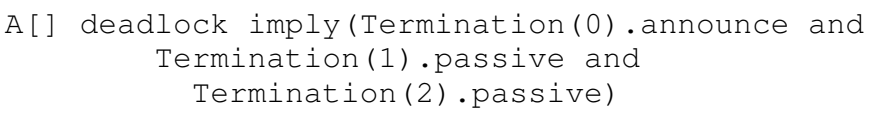

The requirement $\mathrm{R} 2$ says that the system is terminated properly if the leader reaches at announce state for every path of execution. The formula for the requirement is given as:

$$
\text { A }<>\text { Termination }(0) \text {. announce }
$$

All the processes must reach at passive state for proper termination of the system according to the requirement R3. Given below is the formula for R3:

$$
\text { E<> forall(i:id_t) Termination(i).passive }
$$

The formula for INV1 is presented below. A process moves from passive to A2_l state after receiving a basic message. Then this process updates its weight in next transition. It means, like passive state this process has a zero weight at $A 2 \_1$ state. So, we are including this state in the formula for INV1.

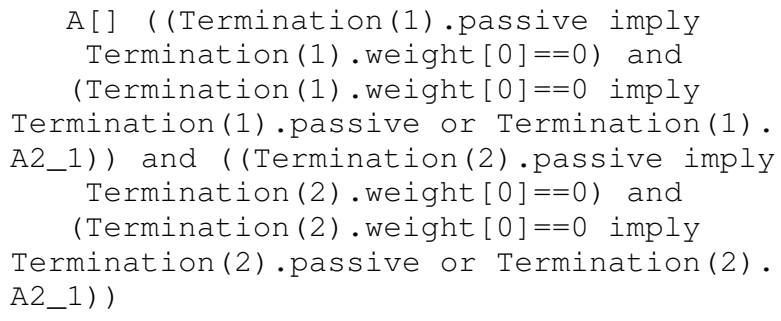

Now we discuss the formula for INV2. Each process updates two global variables $\mathrm{W} 1$ and $\mathrm{W} 2$ after receiving a basic or a control message. The $\mathrm{W} 1$ records the first value and $\mathrm{W} 2$ records the second value of received weight. If these variables always keep some nonzero value of weight then it means every message sent by any process has a non zero weight. The formula for this invariant is given as:

$$
\text { A [ ] } W 1 !=0 \text { and } W 2 !=0
$$

The invariant INV3 is for all healthy processes. The formula for this invariant is:

\author{
A[] forall(i:id_t) \\ Termination.In_Out_Equal $(i)==$ true
}

This invariant INV3 uses five functions for its calculations. These functions are AddInWeights(), InSum(), AddOutWeights(), OutSum(), and In_Out_Equal(). All these functions perform combined functionality for verification of INV3. The function AddInWeights() adds all the incoming weights recorded in In_arr[] of calling process. The function $\operatorname{InSum}()$ adds the current weight and the some of incoming weights and stores the result in tempIn[]. The AddOutWeights() function adds all the outgoing weights recorded in Out arr [] of a process. The function OutSum() calculates the sum of initial weight and all outgoing weights of a process and stores the result in tempOut[]. At the end In_Out_Equal() checks the equality of tempIn[] and tempOut[]. If both arrays are equal then this function returns a true value otherwise a false value.

\section{B. Requirement Formal Specification for Model2}

According to requirement $\mathrm{R} 1$, some process reaches at the announce at some time. The formula for this requirement is given as:

$$
\text { A }<>\text { exists(i:id_t) Termination(i).announce }
$$

The requirements R2 and R3 are clearly discussed with examples in Model2 requirements part. Now we discuss the formula for invariant INV1. Formalism for this invariant is given as:

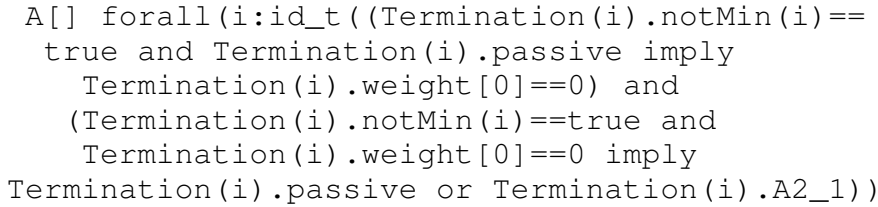

The function notMin() checks if the calling process belongs to faulty set of processes or it is a healthy process with minimum id. It returns false if the process is faulty or it is healthy with minimum id. It returns true otherwise allowing other processes to check their weight at the passive state.

The formalism for INV3 for Model2 is similar to formalism of INV3 for Model1. All the functions and their definitions are same. But Model2 uses an extra function Equal() that checks if the calling process is faulty. It means we are just concerned with the calculations for healthy processes. If calling process is healthy then it returns true if the invariant is preserved and returns false if the invariant is violated. The formula for this invariant is given as:

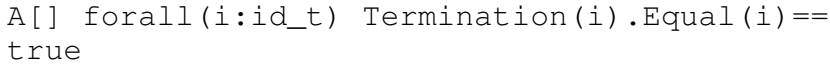

\section{VERIFICATION RESULTS FOR MODEL1}

This section shows the simulation results of formalism for functional requirements and invariants for Model1. These results are collected by executing the formulas in verifier of the UPPAAL toolset. For simplicity we use the Buffer instead of the MessageBuffer in all counterexamples. Results for Model1 are given below in Table VIII. We verify our system model for,

Total Number of Termination Process Instances $=3$

Total Weight of the System $=1$ 
TABLE VIII. REQUIREMENT RESUlts FOR MODEL1

\begin{tabular}{|l|l|l|}
\hline \hline Requirement & Status & Computational Time \\
\hline \hline R1* & Satisfied, 225504590 states & $57600 \mathrm{~m} 23.345 \mathrm{~s}$ \\
\hline R2* & Satisfied, 32045480 states & $7200 \mathrm{~m} 53.167 \mathrm{~s}$ \\
\hline R3 & Satisfied, 18986 states & $3.564 \mathrm{~s}$ \\
\hline INV1* & Satisfied, 28006031 states & $7210 \mathrm{~m} 34.453 \mathrm{~s}$ \\
\hline INV2* & Satisfied, 25933265 states & $7206 \mathrm{~m} 12.560 \mathrm{~s}$ \\
\hline INV3* & Satisfied, 20568945 states & $7002 \mathrm{~m} 19.350 \mathrm{~s}$ \\
\hline
\end{tabular}

\section{Weight of Each Instance $=1 / 3$}

Requirement R3 is satisfied. For R1, R2, INV1, INV2 and INV3, state space is not fully explored. We are not sure about their final results. We explored these requirements for breadth first searching technique. Our claims are limited to the number of states and time given in Table VIII.

\section{VerificATION RESUlts FOR MODEl2}

Results for Model2 are given below in Table IX. We verify our system model for,

Total Number of Termination Process Instances = 4

Total Weight of the System $=1$

Weight of Each Instance $=1 / 4$

TABLE IX. REQUiREMENT RESUlts FOR MOdel2

\begin{tabular}{|l|l|l|}
\hline Requirement & Status & Computational Time \\
\hline \hline R1 & Not satisfied & $06.307 \mathrm{~s}$ \\
\hline INV1* & Satisfied, 25166435 states & $7215 \mathrm{~m} 33.873 \mathrm{~s}$ \\
\hline INV2* & Satisfied, 42542339 states & $11520 \mathrm{~m} 21.212 \mathrm{~s}$ \\
\hline INV3 & Not satisfied & $20.353 \mathrm{~s}$ \\
\hline
\end{tabular}

In Model2, for INV1 and INV2, state space is not fully explored. We are not sure about the final results of these invariants. We explored these requirements using breadth first searching technique and we claim these results to the number of states given in Table IX. The requirement $R 1$ is not satisfied. The counterexample for this violation is shown in Fig. 11. The $p 3$ sends a basic message to $p 0$. The $p 1$ and $p 2$ send control messages to $p 0$. Then $p 0$ sends a basic message to $p 3$. After this, $p 3$ receives the basic message sent by $p 0$ and sends the control message to $p 0$. After doing all these communications, $p 0$ fails and other three processes become passive. In this case, there is no process that is active and detect $p 0$ as faulty. So, it is not possible to collect the weights carried by $p 0$. Therefore, the announce state is not reachable in this scenario.

The invariant INV3 violates in the given scenario. The $p 0$ sends a basic message to $p 1$ and becomes passive. The $p 1$ sends a basic message to $p 0$ and reaches at $A 1$ state. The $p 2$ sends a control message to $p O$ and visits $A 3$ state. After this, $p O$ receives the control message of $p 0$ and becomes passive. At passive state, it receives the basic message from $p l$ and reaches at active state. In the whole activity p3 stays at active state. The invariant is not satisfied in this situation. The counter example for this violation is shown in Fig. 12.

\section{Limitations And Challenges}

There are some limitations for verification of intended termination detection protocol. We limit the concurrent termination processes to three in case of fault-free distributed system and four for faulty distributed system. The basic message sending limit for any process is two. The models generate a huge state space with millions of states. The purpose of these limitations is to reduce the state space of our computations. The machines and servers used in our verification have limited resources for memory and speed. We performed some computations on the machine with 16GB RAM, core i7(4th Gen) CPU and $3.4 \mathrm{GHz}$ clock speed. We also performed some computations on mammoth server (tue.nl) which has 56 machines with $2 \mathrm{GHz}$ speed and a total of 935GB RAM.

We faced some challenges during the modeling and verification of the protocol. The total weight of the system is 1 and initial weight of each process is $1 / n$ where $n$ is the total number of processes. The expression $1 / n$ returns fractional values. At the end of the computation it becomes hard to make the sum of all weights to 1 due to possible rounding errors. The first challenge was to manage a single weight in the form of a pair of integers. We presented the weight $1 / n$ in the form of two values as $[1, n]$. This method created many difficulties for accurately calculating the incoming, outgoing and current weights of the processes. This was also a big challenge to correctly model the protocol and its invariants in UPPAAL. We used model abstraction for reducing the state space that was also a challenge.

\section{CONCLUSION}

We formalised both parts of termination detection protocol as specified in [7] in the timed-automata-theoretic formalism of UPPAAL. We formally specified and performed verification analysis of the protocol with respect to its functional requirements and invariants. During our formal analysis, we found some scenarios in which the protocol does not meet its functional requirements. Counter example are there to witness the claimed results.

\section{REFERENCES}

[1] W. Fokkink, Distributed algorithms: an intuitive approach. MIT Press, 2013.

[2] K. M. Chandy and J. Misra, "Asynchronous distributed simulation via a sequence of parallel computations," Commun. ACM, vol. 24, no. 4, pp. 198-206, Apr. 1981. [Online]. Available: http://doi.acm.org/10.1145/358598.358613

[3] K. M. Chandy, J. Misra, and L. M. Haas, "Distributed deadlock detection," ACM Trans. Comput. Syst., vol. 1, no. 2, pp. 144-156, May 1983. [Online]. Available: http://doi.acm.org/10.1145/357360.357365

[4] G. Tel and F. Mattern, "The derivation of distributed termination detection algorithms from garbage collection schemes," ACM Trans. Program. Lang. Syst., vol. 15, no. 1, pp. 1-35, Jan. 1993. [Online]. Available: http://doi.acm.org/10.1145/151646.151647

[5] E. W. Dijkstra and C. Scholten, "Termination detection for diffusing computations," Information Processing Letters, vol. 11, no. 1, pp. 1-4, 1980. [Online]. Available: http://www.sciencedirect.com/science/article/pii/0020019080900216

[6] N. Francez, "Distributed termination," ACM Trans. Program. Lang. Syst., vol. 2, no. 1, pp. 42-55, Jan. 1980. [Online]. Available: http://doi.acm.org/10.1145/357084.357087

[7] Y. Tseng, "Detecting termination by weight-throwing in a faulty distributed system," J. Parallel Distrib. Comput., vol. 25, no. 1, pp. 7-15, 1995. [Online]. Available: https://doi.org/10.1006/jpdc.1995.1025

[8] X. Wang and J. Mayo, "A general model for detecting distributed termination in dynamic systems," in 18th International Parallel and Distributed Processing Symposium, 2004. Proceedings., April 2004, p. 84.

[9] G. Tel and F. Mattern, "The derivation of distributed termination detection algorithms from garbage collection schemes," ACM Trans. Program. Lang. Syst., vol. 15, no. 1, pp. 1-35, Jan. 1993. [Online]. Available: http://doi.acm.org/10.1145/151646.151647

[10] F. Mattern, H. Mehl, A. A. Schoone, and G. Tel, "Global virtual time approximation with distributed termination detection algorithms," Tech. Rep., 1991.

[11] S. Chandrasekaran and S. Venkatesan, "A message-optimal algorithm for distributed termination detection," J. Parallel Distrib. Comput., vol. 8, no. 3, pp. 245-252, $\operatorname{mar}$ 1990. [Online]. Available: http://dx.doi.org/10.1016/0743-7315(90)90099-B

[12] J. Pang, Analysis of a Security Protocol in $\mu C R L$, C. George and H. Miao, Eds. Berlin, Heidelberg: Springer Berlin Heidelberg, 2002. [Online]. Available: http://dx.doi.org/10.1007/3-540-36103- $0_{4} 0$ 


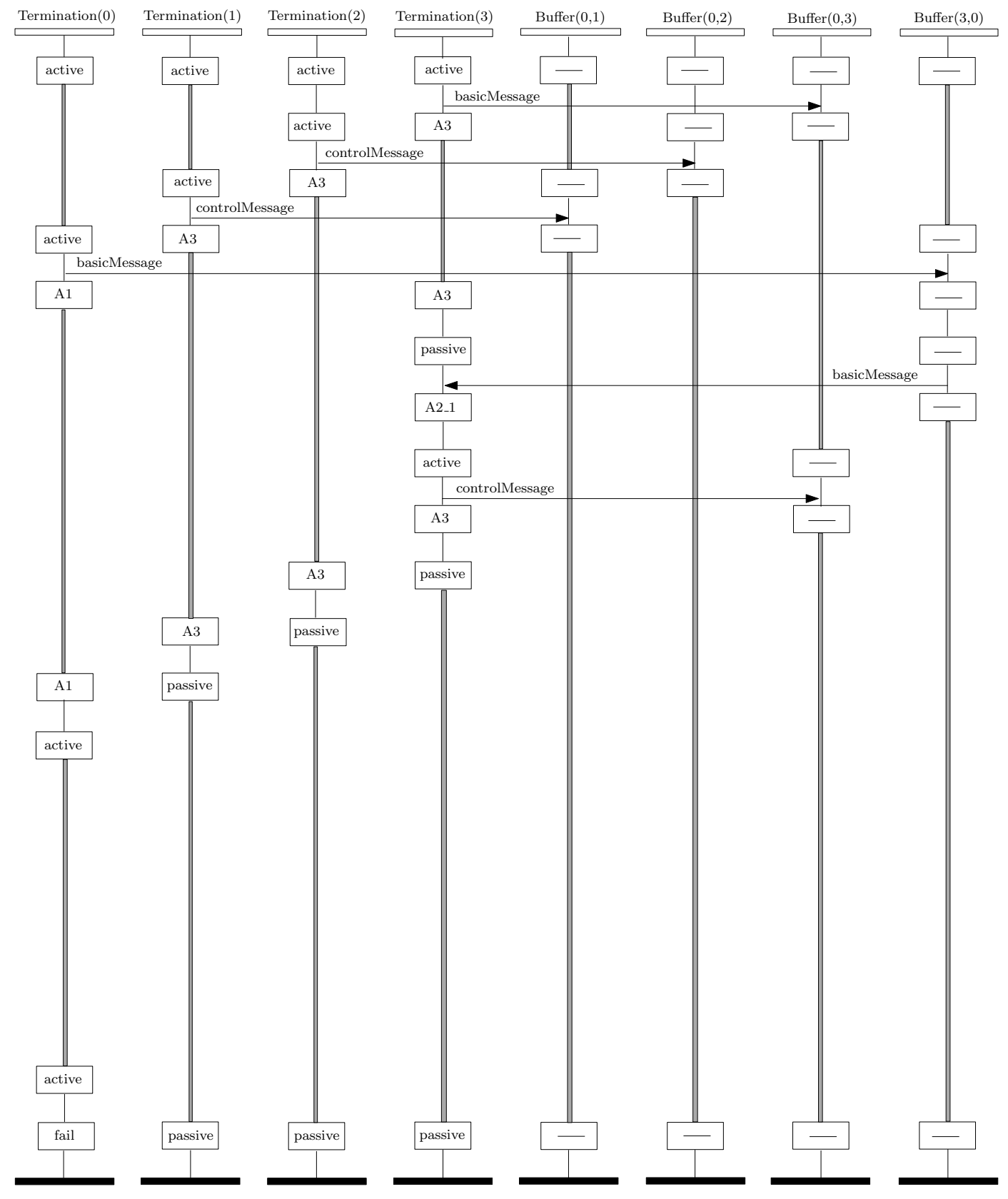

Fig. 11. Trace for R1 in Model2.

[13] W. H. J. Feijen and A. J. M. van Gasteren, Shmuel Safra's Termination Detection Algorithm. New York, NY: Springer New York, 1999. [Online]. Available: http://dx.doi.org/10.1007/978-1-4757-3126- $2{ }_{2} 9$

[14] N. Mittal, S. Venkatesan, and S. Peri, Message-Optimal and Latency-Optimal Termination Detection Algorithms for Arbitrary Topologies, R. Guerraoui, Ed. Berlin, Heidelberg: Springer Berlin Heidelberg, 2004. [Online]. Available:19] http://dx.doi.org/10.1007/978-3-540-30186- ${ }_{2} 1$

[15] N. R. Mahapatra and S. Dutt, "An efficient delay-optimal distributed termination detection algorithm," Journal of Parallel and Distributed Computing, vol. 67, no. 10, pp. 1047-1066, 2007. [Online]. Available: http://www.sciencedirect.com/science/article/pii/S0743731507000998

[16] J. S. Ostroff, "Formal methods for the specification and design of real-time safety critical systems," J. Syst. Softw., vol. 18, no. 1, pp. 33-60, Apr. 1992. [Online]. Available: http://dx.doi.org/10.1016/0164-1212(92)90045-L

[17] A. O. Gomes and M. V. M. Oliveira, Formal Specification of a Cardiac Pacing System, A. Cavalcanti and D. R. Dams, Eds. Berlin, Heidelberg: Springer
Berlin Heidelberg, 2009. [Online]. Available: http://dx.doi.org/10.1007/9783-642-05089-3 44

18] Y. K. H. Lau, "The design of distributed safety critical software using csp," in IEE Colloquium on Safety Critical Software in Vehicle and Traffic Control, Feb 1990, pp. 8/1-8/5.

K. G. Larsen, P. Pettersson, and W. Yi, "Uppaal in a nutshell," 1997. 


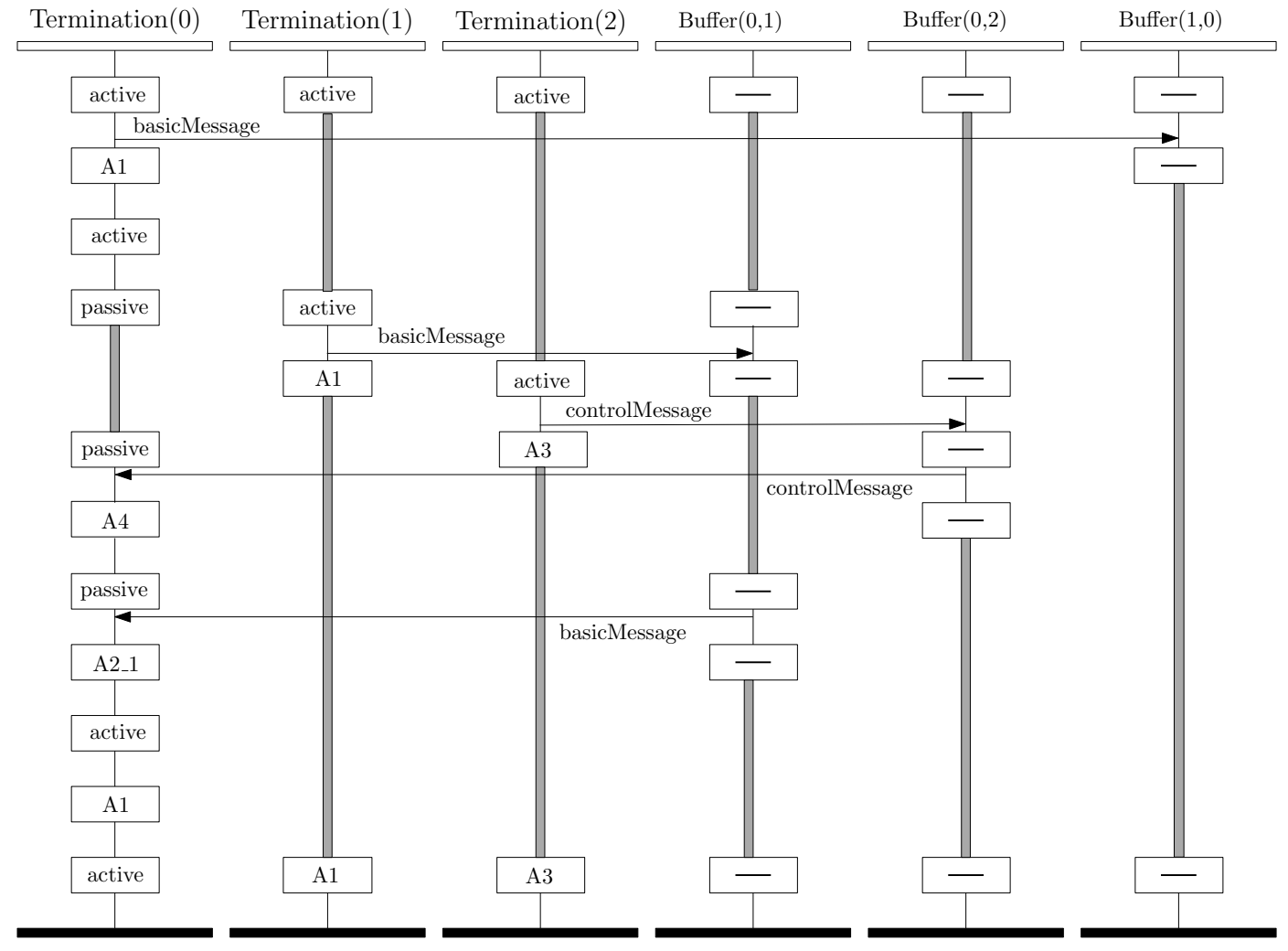

Fig. 12. Trace for INV3 in Model2. 